\title{
Bayesian Group Chain Sampling Plan for Poisson Distribution with Gamma Prior
}

\author{
Waqar Hafeez ${ }^{1}$, Nazrina Aziz ${ }^{1,2, *}$, Zakiyah Zain ${ }^{1,2}$ and Nur Azulia Kamarudin ${ }^{1}$ \\ ${ }^{1}$ School of Quantitative Sciences, Universiti Utara Malaysia, UUM Sintok, 06010, Kedah, Malaysia \\ ${ }^{2}$ Institute of Strategic Industrial Decision Modelling (ISIDM), Universiti Utara Malaysia, UUM Sintok, \\ 06010, Kedah, Malaysia \\ *Corresponding Author: Nazrina Aziz. Email: nazrina@uum.edu.my \\ Received: 22 April 2021; Accepted: 07 June 2021
}

\begin{abstract}
Acceptance sampling is a statistical quality control technique that consists of procedures for sentencing one or more incoming lots of finished products. Acceptance or rejection is based on the inspection of sampled products drawn randomly from the lot. The theory of previous acceptance sampling was built upon the assumption that the process from which the lots are produced is stable and the process fraction nonconforming is a constant. Process variability is inevitable due to random fluctuations, which may inadvertently lead to quality variation. As an alternative to traditional sampling plans, Bayesian approach can be used by considering prior information of the process. Using different combinations of design parameters, this study introduces a Bayesian group chain sampling plan (BGChSP). For the first time in group chain sampling plan, the probability of lot acceptance is derived by using Poisson distribution to estimate an average number of defectives. Gamma distribution is used as a prior distribution with Poisson distribution. Taking into account both consumer's and producer's risks, this research considers two quality regions namely, probabilistic quality region (PQR) and indifference quality region (IQR). By minimizing consumer's and producer's risks, BGChSP can be used to minimize the average number of defective products in industry.
\end{abstract}

Keywords: Bayesian; acceptance sampling; gamma; produce risk; consumer risk

\section{Introduction}

There is tough competition in the industry by rapidly increasing the needs of statistical and analytical techniques towards the improvement of product quality [1-3]. This study is related to Bayesian group chain sampling plan (BGChSP) by using a novel approach called quality region. Instead of considering a point estimate of the quality of a process, this plan is based on a range of quality levels. The producer's risk and consumer's risk are associated with every sampling plan [4]. A good plan delivers decision rules of acceptance for both producer and consumer to meet the present quality condition of the product [5]. Improvement in technology

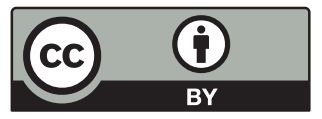

This work is licensed under a Creative Commons Attribution 4.0 International License, which permits unrestricted use, distribution, and reproduction in any medium, provided the original work is properly cited. 
is rapidly increasing with the passage of time and supplier needs high-quality products with low defective fraction [6]. Unfortunately, in some situations, traditional methods cannot detect the defective product with such quality level. Thus, quality regions were introduced to overcome such problems [7,8]. By involving probabilistic quality region (PQR) and indifference quality region (IQR), this study designs the parameters for the plan indexed with quality region.

Bayesian approach is based on the combination of current and prior information for the selection of distribution. In acceptance sampling plan, Bayesian approach is required to specify a distribution of defectives from lot to lot [7]. Prior distribution is the expected distribution of the lot quality, that is under inspection. Prior distribution is formulated before taking the sample and empirical knowledge is based on the sample under study called the distribution of sample or data. The combination of prior and empirical information leads to a decision about the current lot either it will be accepted or rejected.

\section{Literature Review}

Epstein [9] was the first who introduced the idea of single sampling plan (SSP), to distinguish between good and bad lot for zero and one acceptance number. Dodge [10] introduced the chain sampling plan to overcome the deficiency of SSP, with more than one acceptance number and for the general family of chain sampling inspection plan [11]. Under an assumption that cost is linear in $p$ that is the fraction of defectives. Hald [12] provides a system of attribute single sampling plan obtained by minimizing average cost. Later, single sampling plan was modified by using acceptable quality level (AQL) and limiting quality level (LQL) for Bayesian single sampling plan with weighted Poisson distribution [13] and Poisson distribution [14]. Later, in the construction and performance measure, a Bayesian chain sampling plan was discussed for gamma prior [15]. For Beta prior, a Bayesian double sampling plan was considered for the construction and performance measure through quality region [16]. Mathew et al. [17] also work in two-sided complete Bayesian chain sampling plan with Poisson and gamma as prior distribution.

Aslam et al. [18] developed a group sampling plan (GSP), by considering multiple testers at the same time. In GSP the sample size is divided into $g$ number of groups based on the available number of testers $n=r * g$. Aslam et al. [19] designed group acceptance sampling plan (GASP) for truncated life tests, with the product lifetime following Pareto distribution of the second kind. Later, for a family of Pareto distribution, an efficient GASP was introduced by Mughal et al. [20]; they assumed the total number of defectives as groups. The number of defectives in the proposed plan was recorded based on all groups instead of an individual group. Moreover, for a family of Pareto distribution, an economic reliability acceptance sampling plan was proposed for an efficient group sampling technique [21]. By satisfying design parameters for a given group and acceptance number, a minimum termination time was required for the proposed plan. Using GASP for Pareto distribution of 2nd kind, an economic reliability group acceptance sampling plan was developed by Mughal et al. [22]. For the biased data theory to find the required design parameters, they used Poisson and weighted Poisson distributions. It was proven that their proposed plans required minimum testing time.

Mughal et al. [23] developed a GChSP plan for the lifetime of a product following Pareto distribution of 2nd kind. Satisfying pre-assumed design parameters at several quality levels, probability of lot acceptance was obtained. Based on their sampling plan, Mughal [24] extended and proposed a generalized GChSP. By considering several values of the proportion of defectives, the minimum sample size and probability of lot acceptance were found to satisfy pre-specified 
consumer's risk. Recently, Hafeez et al. [25] also worked on this plan and estimated AQL and LQL for the average proportion of defective products in their study.

Based on Mughal et al. [23] plan, this study concerns with the development of a Bayesian group chain sampling plan (BGChSP). In group chain sampling plan, the probability of lot acceptance is derived by using Poisson distribution and gamma is used as a prior distribution. For PQR and IQR, this study designs the plan indexed parameters which are: AQL, LQL, producer's risk $\alpha$, consumer's risk $\beta$, for the specified values of the number of testers $r$ and shape parameter $s$. Also, the numerical illustrations are provided for prior distribution parameters.

\section{Methodology}

The operating procedure for the proposed plan is based on the following steps:

(1) Select an ideal number of $g$ groups for each lot and assign $r$ items to each group with the sample size required $n=g * r$.

(2) Count the number of defectives $d$.

(3) If $d=0$, accept the lot.

(4) If $d>1$, reject the lot.

(5) If $d=1$, and no defectives was found in the immediately preceding $i$ samples, then accept the lot.

All the above steps can be summarized in a flow chart presented in Fig. 1.

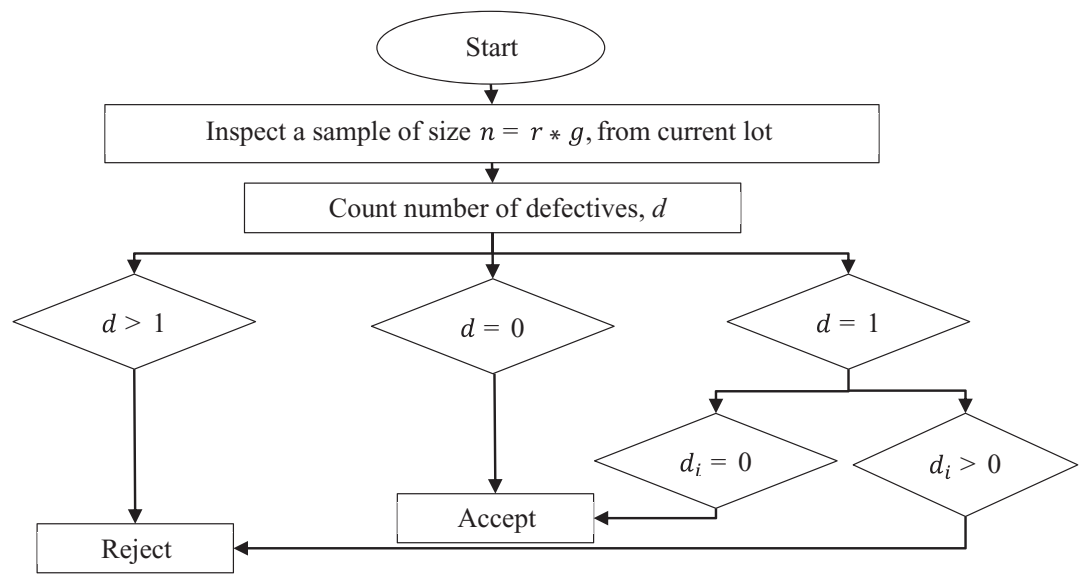

Figure 1: Operating procedure of the proposed sampling plan

In attribute acceptance sampling, lot inspection is based on the number of defectives (discrete variable). The procedure of GChSP for $i=2$, is illustrated through a tree diagram, represented in Fig. 2, where defective and non-defective products are denoted by $D$ and $\bar{D}$, respectively. From Fig. 2, for $i=2$ it can observed that GChSP has five acceptance criteria (AC) $\{D D \bar{D}, D \overline{D D}, \bar{D} D \bar{D}, \overline{D D} D, \overline{D D D}\}$. Here $\{D D \bar{D}, D \overline{D D}, \bar{D} D \bar{D}, \overline{D D D}\}$ concern with the current lot, hence the probability of lot acceptance is $P_{0}$ and $\{\overline{D D} D\}$ has a probability of lot acceptance $P_{0} P_{0} P_{1}$. Finally in the group chain, the probability of lot acceptance can be written as:

$L(p)_{G C h S P}=P_{0}+P_{0} P_{0} P_{1}$ 


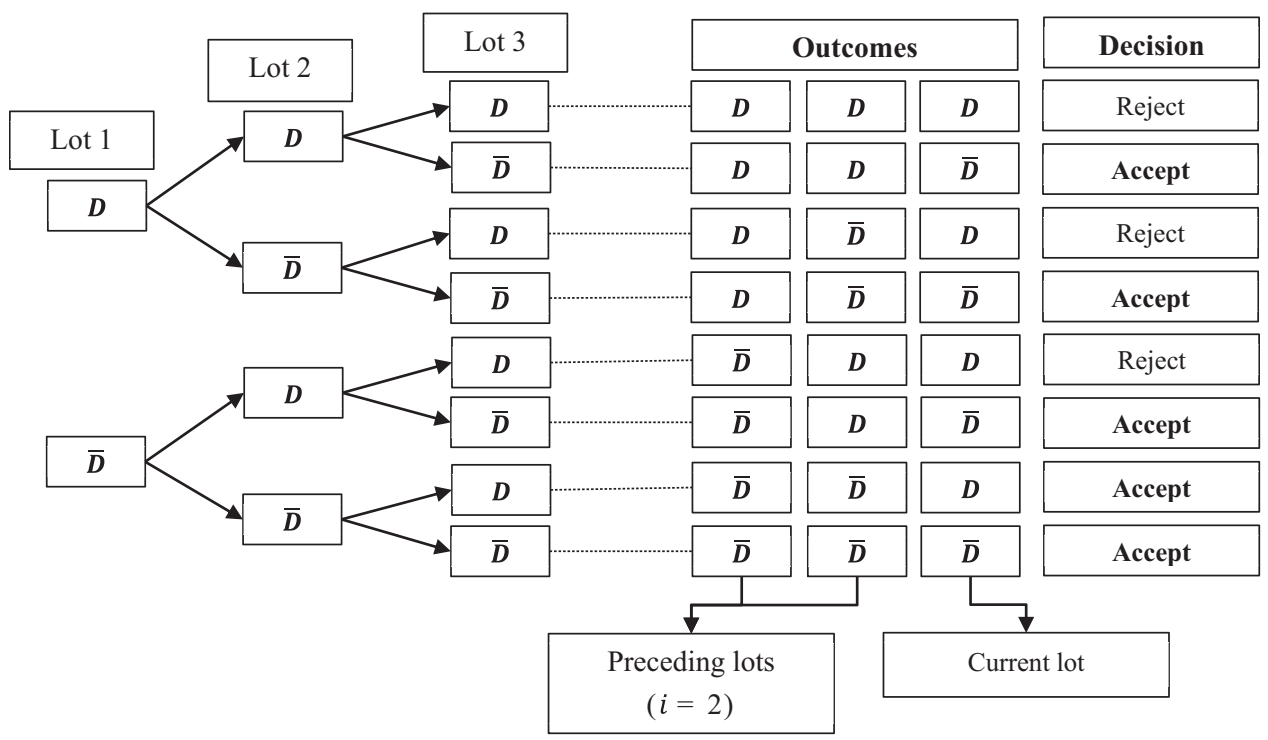

Figure 2: Tree diagram for the proposed sampling plan

$L(p)_{G C h S P}=P_{0}+P_{1}\left(P_{0}\right)^{2}$

The general expression for $i=2$ is like the operating characteristics (OC) function of ChSP-1 given by Dodge [10].

$L(p)_{G C h S P}=P_{0}+P_{1}\left(P_{0}\right)^{i}$

For the average number of defectives, consider Poisson distribution with mean $\mu=n p$ and $n=r * g$, to achieve the probability of lot acceptance for zero and one defective product which are respectively:

$P_{0}=e^{-(r * g) p}$,

$P_{1}=(r * g) p e^{-(r * g) p}$.

After replacing Eqs. (4) and (5) in Eq. (3), we get:

$L(p)_{G C h S P}=e^{-(r * g) p}\left\{1+(r * g) p e^{-(r * g) p i}\right\}$.

Let us consider gamma distribution as a suitable prior for the Poisson distribution [26,27], with pdf:

$f(p)=\frac{t^{s}}{\Gamma(s)} p^{s-1} e^{-t p}$,

where shape parameter, $s>0$ and rate parameter, $t>0$ with mean $\mu=\frac{s}{t}$ under the proposed sampling plan. For the average probability of lot acceptance, the general expression used in Bayesian is as follows:

$\bar{P}=\int_{0}^{\infty} L(p) f(p) d p$. 
After replacing Eqs. (6) and (7) in Eq. (8) and then from simplification we get:

$\bar{P}=\frac{t^{s}}{\Gamma(s)}\left[\frac{\Gamma(s)}{(r * g+t)^{s}}+(r * g) \frac{\Gamma(s+1)}{(r * g *(1+i)+t)^{s+1}}\right]$,

$\bar{P}=\left(\frac{t}{r * g+t}\right)^{s}+(r * g) \frac{s t^{s}}{(r * g(1+i)+t)^{s+1}}$.

Upon substituting mean $\mu=\frac{s}{t}$ in Eq. (10) and simplifying:

$\bar{P}=\left(\frac{s}{r g \mu+s}\right)^{s}+\frac{r g \mu s^{s+1}}{(\operatorname{rg} \mu(1+i)+s)^{s+1}}$.

Further simplification of Eq. (11), for $s=1,2,3$ gives:

$\bar{P}=\frac{1}{(r g \mu+1)}+\frac{r g \mu}{(\operatorname{rg} \mu(1+i)+1)^{2}}$,

$\bar{P}=\frac{4}{(r g \mu+2)^{2}}+\frac{8 r g \mu}{(\operatorname{rg} \mu(1+i)+2)^{3}}$,

$\bar{P}=\frac{27}{(\operatorname{rg} \mu+3)^{3}}+\frac{81 \operatorname{rg} \mu}{(\operatorname{rg} \mu(1+i)+3)^{4}}$.

It is to be noted that Newton's approximation is employed in Eqs. (12)-(14) to find the quality regions of BGChSP, where $\mu$ is used as a point of control by reducing $\bar{P}$. Tab. 1 presents the average number of defectives for the specified values of shape parameter $s=1,2,3$; the number of testers $r=2,3,4$ and number of preceding lots $i=1,2,3,4$. Operating ratios for different values of consumer's risk and producer's risk are presented in Tab. 2.

Example 1: In Tab. 1, for $s=1, r=2, i=3$ and $\bar{P}=0.50$ the corresponding value of the average number of defectives is 0.5788 and for $s=2, r=3$ and $i=4$, the corresponding value is 0.3017. From Tab. 1, it can be concluded that as the values of $s, r$ and $i$ are increased the average number of defective products decreases.

\subsection{Designing Sampling Plans for Given $A Q L$ and $L Q L$}

For the selection of BGChSP, Tabs. 1-2 are used for specified AQL, LQL, $\alpha$ and $\beta$ by using the following three steps: (i) construct a plan for the given points (AQL, $1-\alpha$ ) and (LQL, $\beta$ ), then compute the desired operating ratio; (ii) from Tab. 2, find the value of the operating ratio that is equal to or just less than the desired operating ratio in the desired columns for $\alpha, \beta$; (iii) obtain the corresponding values of $s, r, g$ and $i$, from Tab. 1 for the corresponding located value of the operating ratio given in Tab. 2.

\subsection{Construction of Quality Regions}

\subsubsection{Probabilistic Quality Region (PQR)}

In this quality region, the product is accepted with the maximum probability of 0.95 and minimum probability of 0.10 , where 0.95 corresponds to (AQL, $1-\alpha$ ) and 0.10 corresponds to (LQL, $\beta$ ). In other words, $\mathrm{PQR}\left(R_{1}\right)$ is exactly the conventional setting of $\mathrm{AQL}=\mu_{1}$ and $\mathrm{LQL}=$ $\mu_{2}$. In Fig. 3, we can see the PQR lies between $\mu_{1}<R_{1}<\mu_{2}$. 
Table 1: For specified values of $s, r, i$ and $\bar{P}$, the average number of defectives for BGChSP

\begin{tabular}{|c|c|c|c|c|c|c|c|c|c|c|}
\hline & $r$ & $i$ & 0.99 & 0.95 & 0.9 & 0.5 & 0.25 & 0.1 & 0.05 & 0.01 \\
\hline \multirow[t]{12}{*}{1} & 2 & 1 & 0.0323 & 0.0843 & 0.1363 & 0.7339 & 1.9938 & 5.7479 & 11.9990 & 62.0005 \\
\hline & 2 & 2 & 0.0253 & 0.067 & 0.1096 & 0.6247 & 1.7383 & 5.0722 & 10.6279 & 55.0740 \\
\hline & 2 & 3 & 0.0216 & 0.0581 & 0.0963 & 0.5788 & 1.6408 & 4.8277 & 10.1401 & 52.6412 \\
\hline & 2 & 4 & 0.0193 & 0.0526 & 0.0881 & 0.5546 & 1.5931 & 4.7121 & 9.9119 & 51.5148 \\
\hline & 3 & 1 & 0.0215 & 0.0562 & 0.0908 & 0.4892 & 1.3292 & 3.8319 & 7.9994 & 41.3347 \\
\hline & 3 & 2 & 0.0168 & 0.0447 & 0.0731 & 0.4164 & 1.1588 & 3.3814 & 7.0852 & 36.7168 \\
\hline & 3 & 3 & 0.0144 & 0.0388 & 0.0642 & 0.3859 & 1.0939 & 3.2185 & 6.7600 & 35.0959 \\
\hline & 3 & 4 & 0.0128 & 0.0351 & 0.0587 & 0.3697 & 1.0620 & 3.1414 & 6.6079 & 34.3417 \\
\hline & 4 & 1 & 0.0161 & 0.0422 & 0.0681 & 0.3669 & 0.9969 & 2.8739 & 5.9995 & 31.0002 \\
\hline & 4 & 2 & 0.0126 & 0.0335 & 0.0548 & 0.3123 & 0.8691 & 2.5361 & 5.3140 & 27.5374 \\
\hline & 4 & 3 & 0.0108 & 0.0291 & 0.0481 & 0.2894 & 0.8204 & 2.4139 & 5.0700 & 26.3206 \\
\hline & 4 & 4 & 0.0096 & 0.0263 & 0.0440 & 0.2773 & 0.7965 & 2.3561 & 4.9560 & 25.7573 \\
\hline \multirow[t]{12}{*}{2} & 2 & 1 & 0.0363 & 0.0914 & 0.1429 & 0.6055 & 1.2760 & 2.5818 & 4.0480 & 10.2300 \\
\hline & 2 & 2 & 0.0284 & 0.0722 & 0.1141 & 0.5123 & 1.1180 & 2.3188 & 3.6742 & 9.4007 \\
\hline & 2 & 3 & 0.0242 & 0.0624 & 0.0995 & 0.4730 & 1.0615 & 2.2367 & 3.5647 & 9.1760 \\
\hline & 2 & 4 & 0.0215 & 0.0561 & 0.0905 & 0.4526 & 1.0361 & 2.2032 & 3.5219 & 9.0921 \\
\hline & 3 & 1 & 0.0242 & 0.0609 & 0.0953 & 0.4037 & 0.8506 & 1.7212 & 2.6987 & 6.8200 \\
\hline & 3 & 2 & 0.0189 & 0.0482 & 0.0761 & 0.3415 & 0.7454 & 1.5458 & 2.4494 & 6.2671 \\
\hline & 3 & 3 & 0.0161 & 0.0416 & 0.0663 & 0.3153 & 0.7077 & 1.4911 & 2.3765 & 6.1173 \\
\hline & 3 & 4 & 0.0143 & 0.0374 & 0.0603 & 0.3017 & 0.6907 & 1.4688 & 2.3479 & 6.0614 \\
\hline & 4 & 1 & 0.0182 & 0.0457 & 0.0715 & 0.3027 & 0.6380 & 1.2909 & 2.0240 & 5.1149 \\
\hline & 4 & 2 & 0.0142 & 0.0361 & 0.0571 & 0.2561 & 0.5590 & 1.1594 & 1.8371 & 4.7002 \\
\hline & 4 & 3 & 0.0121 & 0.0312 & 0.0498 & 0.2365 & 0.5308 & 1.1184 & 1.7823 & 4.5880 \\
\hline & 4 & 4 & 0.0108 & 0.0281 & 0.0452 & 0.2263 & 0.5180 & 1.1016 & 1.7609 & 4.5461 \\
\hline \multirow[t]{12}{*}{3} & 2 & 1 & 0.0381 & 0.0946 & 0.1461 & 0.5690 & 1.1049 & 2.0096 & 2.8989 & 5.9575 \\
\hline & 2 & 2 & 0.0298 & 0.0746 & 0.1164 & 0.4798 & 0.9698 & 1.8209 & 2.6659 & 5.5848 \\
\hline & 2 & 3 & 0.0253 & 0.0643 & 0.1012 & 0.4421 & 0.9234 & 1.7682 & 2.6076 & 5.5052 \\
\hline & 2 & 4 & 0.0225 & 0.0577 & 0.0917 & 0.4226 & 0.9037 & 1.7492 & 2.5881 & 5.4810 \\
\hline & 3 & 1 & 0.0254 & 0.0631 & 0.0974 & 0.3794 & 0.7366 & 1.3397 & 1.9326 & 3.9717 \\
\hline & 3 & 2 & 0.0198 & 0.0498 & 0.0776 & 0.3199 & 0.6465 & 1.2139 & 1.7773 & 3.7232 \\
\hline & 3 & 3 & 0.0169 & 0.0429 & 0.0674 & 0.2947 & 0.6156 & 1.1788 & 1.7384 & 3.6702 \\
\hline & 3 & 4 & 0.0150 & 0.0385 & 0.0611 & 0.2817 & 0.6024 & 1.1661 & 1.7254 & 3.6540 \\
\hline & 4 & 1 & 0.0191 & 0.0473 & 0.0731 & 0.2845 & 0.5525 & 1.0048 & 1.4494 & 2.9788 \\
\hline & 4 & 2 & 0.0149 & 0.0373 & 0.0582 & 0.2399 & 0.4849 & 0.9104 & 1.3329 & 2.7924 \\
\hline & 4 & 3 & 0.0127 & 0.0321 & 0.0506 & 0.2210 & 0.4617 & 0.8841 & 1.3038 & 2.7526 \\
\hline & 4 & 4 & 0.0113 & 0.0289 & 0.0459 & 0.2113 & 0.4518 & 0.8746 & 1.2941 & 2.7405 \\
\hline
\end{tabular}

The range of probability is $R_{1}=\mu_{2}-\mu_{1}$ which is derived from the equation of the average probability of acceptance Eq. (11). This Eq. (11) is used in the same way to find AQL, LQL and operating ratios represented in Tab. 3. 
Table 2: For specified $\alpha$ and $\beta$ in BGChSP, operating ratio $\mu_{2} / \mu_{1}$ values are tabulated against $s, r$ and $i$

\begin{tabular}{|c|c|c|c|c|c|c|c|c|c|c|c|}
\hline & \multirow[t]{2}{*}{$r \quad i$} & $i$ & \multicolumn{3}{|l|}{$\alpha=0.01$} & \multicolumn{3}{|l|}{$\alpha=0.05$} & \multicolumn{3}{|l|}{$\alpha=0.10$} \\
\hline & & & $=0.01$ & $\beta=0.05$ & $=0.10$ & $\beta=0.01$ & $=0.05$ & $\beta=0.10$ & $\beta=0.01$ & $=0.05$ & $\beta=0.10$ \\
\hline \multirow{12}{*}{\multicolumn{2}{|c|}{$\begin{array}{l}2 \\
2 \\
2 \\
3 \\
3 \\
3 \\
3 \\
4 \\
4 \\
4 \\
4\end{array}$}} & & & & & & & & & & \\
\hline & & & & & & & & & & & \\
\hline & & & & & & & & & & & \\
\hline & & & 2669 & & & & & & & & \\
\hline & & 1 & & & & & & & & & \\
\hline & & & & & & & & & & & \\
\hline & & & & & & & & & & & \\
\hline & & & & & & & & & & & \\
\hline & & & & & & & & & & & \\
\hline & & 2 & & & & & & & & & \\
\hline & & & & & & & & & & & \\
\hline & 4 & & & & & & & & & & \\
\hline \multirow{12}{*}{\multicolumn{2}{|c|}{ 2 }} & 1 & & & & & & & & & \\
\hline & 2 & & & & & & & & & & \\
\hline & 2 & & & & & & & & & & \\
\hline & 2 & 4 & & & & & & & & & \\
\hline & 3 & & & & & & & & & & \\
\hline & 3 & & & & & & & & & & \\
\hline & & & & & & & & & & & \\
\hline & & 4 & & & & & & & & & \\
\hline & & 1 & & & & & & & & & \\
\hline & & & & & & & & & & & \\
\hline & & & & & & & & & & & \\
\hline & 4 & & & & & & & & & & \\
\hline \multirow{12}{*}{3} & 2 & & & & & & & & & & \\
\hline & 2 & & & & & & & & & & \\
\hline & 2 & & & & & & & & & & \\
\hline & 2 & & & & & & & & & & \\
\hline & 3 & & & & & & & & & & \\
\hline & 3 & & & & & & & & & & \\
\hline & 3 & & & & & & & & & & \\
\hline & 3 & & & & & & & & & & \\
\hline & 4 & & & & & & & & & & 56 \\
\hline & 4 & & & & & & & & & & 426 \\
\hline & 4 & & & & & & & & & & \\
\hline & 4 & 4 & 242.5221 & 114.5221 & 77.3982 & 94.827 & 44.7785 & 30.263 & 59.7059 & 28.1939 & 19.0545 \\
\hline
\end{tabular}

3.2.2 Indifference Quality Region (IQR)

In IQR, the product is accepted with maximum and minimum probabilities 0.95 and 0.50 , that correspond to (AQL, $1-\alpha)$ and (LQL, $\beta$ ) respectively. Hence, $R_{0}=\mu_{0}-\mu_{1}$ is the range 
of IQR and lies in the interval $\mu_{1}<R_{0}<\mu_{0}$. This is obtained from the average probability of acceptance Eq. (11).

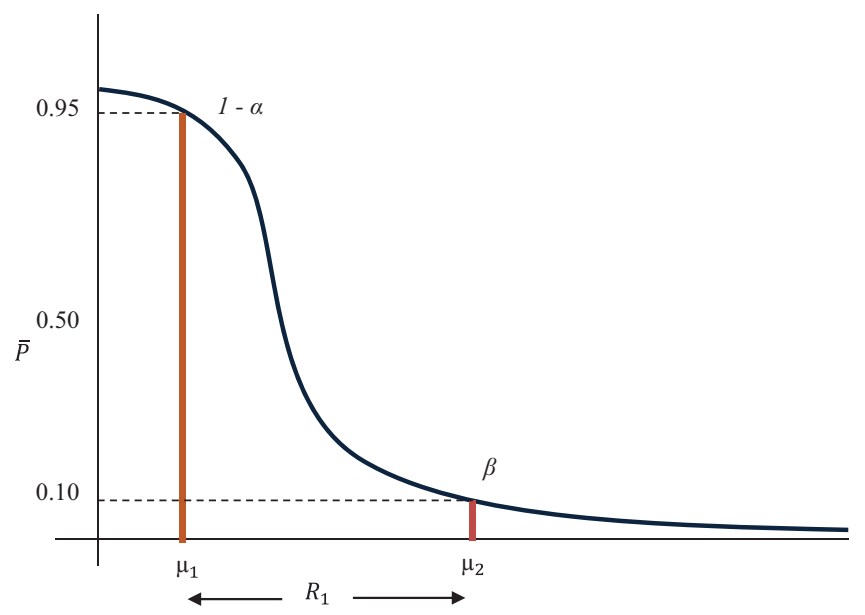

Figure 3: OC curve with pair of coordinates for PQR

Example 2: Let $s=1$ at $\alpha=0.10$ and $\beta=0.05, \mu_{1}=0.008$ and $\mu_{2}=0.90$, then the operating ratio $\frac{\mu_{2}}{\mu_{1}}=112.5$. From Tab. 2 the value 112.6364 with design parameters $s=1, r=4, i=4$ is approximately equal to this calculated operating ratio. With the same design parameters $s=1, r=4$ and $i=4$ from Tab. 1, the corresponding value of $g \mu_{1}=0.044$. The number of groups can be obtained by $\frac{g \mu_{1}}{\mu_{1}}=5.5$-the required minimum number of groups is approximately 6 in order to get the required operating ratio. For this operating ratio, the parametric values are $s=1, g=6$, $r=4, i=4$ at $\alpha=0.10$ and $\beta=0.05$. Also, the corresponding value of AQL is $\mu_{1}=0.008$ and LQL is $\mu_{2}=0.9$.

\subsection{Selection of Sampling Plans}

For different values of $s$ and $r$ in Tab. 3 the operating ratio $T$ is given, i.e., $T=\frac{\mu_{2}-\mu_{1}}{\mu_{0}-\mu_{1}}=\frac{R_{1}}{R_{0}}$, where $R_{1}$ and $R_{0}$ are used to characterize the sampling plan. Operating ratio $T=\frac{R_{1}}{R_{0}}$ can be found for any given values of $\mathrm{PQR}\left(R_{1}\right)$ and IQR $\left(R_{0}\right)$. First we find the value in Tab. 3 under the column of $T$, that is equal to or just less than the specified ratio. Then for this operating ratio, the corresponding parameters values can be determined for $s, r$ and $i$ in BGChSP. 
Table 3: For specified $\mathrm{s}, \mathrm{r}$ and $i$ values of $\mathrm{PQR}, \mathrm{IQR}$ and operating ratio $\mu_{2} / \mu_{1}$

\begin{tabular}{|c|c|c|c|c|c|c|c|c|c|}
\hline$s$ & $r$ & $i$ & $g \mu_{1}$ & $g \mu_{0}$ & $g \mu_{2}$ & $g R_{1}$ & $g R_{0}$ & $T$ & $\mu_{2} / \mu_{1}$ \\
\hline \multirow[t]{12}{*}{1} & 2 & 1 & 0.0843 & 0.7339 & 5.7479 & 5.6636 & 0.6496 & 8.7186 & 68.1839 \\
\hline & 2 & 2 & 0.0670 & 0.6247 & 5.0722 & 5.0052 & 0.5577 & 8.9747 & 75.7045 \\
\hline & 2 & 3 & 0.0581 & 0.5788 & 4.8277 & 4.7696 & 0.5207 & 9.1599 & 83.0929 \\
\hline & 2 & 4 & 0.0526 & 0.5546 & 4.7121 & 4.6595 & 0.5020 & 9.2819 & 89.5837 \\
\hline & 3 & 1 & 0.0562 & 0.4892 & 3.8319 & 3.7757 & 0.4330 & 8.7199 & 68.1833 \\
\hline & 3 & 2 & 0.0447 & 0.4164 & 3.3814 & 3.3367 & 0.3717 & 8.9768 & 75.6465 \\
\hline & 3 & 3 & 0.0388 & 0.3859 & 3.2185 & 3.1797 & 0.3471 & 9.1607 & 82.9510 \\
\hline & 3 & 4 & 0.0351 & 0.3697 & 3.1414 & 3.1063 & 0.3346 & 9.2836 & 89.4986 \\
\hline & 4 & 1 & 0.0422 & 0.3669 & 2.8739 & 2.8317 & 0.3247 & 8.7209 & 68.1019 \\
\hline & 4 & 2 & 0.0335 & 0.3123 & 2.5361 & 2.5026 & 0.2788 & 8.9763 & 75.7045 \\
\hline & 4 & 3 & 0.0291 & 0.2894 & 2.4139 & 2.3848 & 0.2603 & 9.1617 & 82.9519 \\
\hline & 4 & 4 & 0.0263 & 0.2773 & 2.3561 & 2.3298 & 0.2510 & 9.2820 & 89.5856 \\
\hline \multirow[t]{12}{*}{2} & 2 & 1 & 0.0914 & 0.6055 & 2.5818 & 2.4904 & 0.5141 & 4.8442 & 28.2473 \\
\hline & 2 & 2 & 0.0722 & 0.5123 & 2.3188 & 2.2466 & 0.4401 & 5.1048 & 32.1163 \\
\hline & 2 & 3 & 0.0624 & 0.473 & 2.2367 & 2.1743 & 0.4106 & 5.2954 & 35.8446 \\
\hline & 2 & 4 & 0.0561 & 0.4526 & 2.2032 & 2.1471 & 0.3965 & 5.4151 & 39.2727 \\
\hline & 3 & 1 & 0.0609 & 0.4037 & 1.7212 & 1.6603 & 0.3428 & 4.8434 & 28.2627 \\
\hline & 3 & 2 & 0.0482 & 0.3415 & 1.5458 & 1.4976 & 0.2933 & 5.1060 & 32.0705 \\
\hline & 3 & 3 & 0.0416 & 0.3153 & 1.4911 & 1.4495 & 0.2737 & 5.2959 & 35.8438 \\
\hline & 3 & 4 & 0.0374 & 0.3017 & 1.4688 & 1.4314 & 0.2643 & 5.4159 & 39.2727 \\
\hline & 4 & 1 & 0.0457 & 0.3027 & 1.2909 & 1.2452 & 0.2570 & 4.8451 & 28.2473 \\
\hline & 4 & 2 & 0.0361 & 0.2561 & 1.1594 & 1.1233 & 0.2200 & 5.1059 & 32.1163 \\
\hline & 4 & 3 & 0.0312 & 0.2365 & 1.1184 & 1.0872 & 0.2053 & 5.2957 & 35.8462 \\
\hline & 4 & 4 & 0.0281 & 0.2263 & 1.1016 & 1.0735 & 0.1982 & 5.4162 & 39.2029 \\
\hline \multirow[t]{12}{*}{3} & 2 & 1 & 0.0946 & 0.5690 & 2.0096 & 1.915 & 0.4744 & 4.0367 & 21.2431 \\
\hline & 2 & 2 & 0.0746 & 0.4798 & 1.8209 & 1.7463 & 0.4052 & 4.3097 & 24.4089 \\
\hline & 2 & 3 & 0.0643 & 0.4421 & 1.7682 & 1.7039 & 0.3778 & 4.5101 & 27.4992 \\
\hline & 2 & 4 & 0.0577 & 0.4226 & 1.7492 & 1.6915 & 0.3649 & 4.6355 & 30.3154 \\
\hline & 3 & 1 & 0.0631 & 0.3794 & 1.3397 & 1.2766 & 0.3163 & 4.0360 & 21.2314 \\
\hline & 3 & 2 & 0.0498 & 0.3199 & 1.2139 & 1.1641 & 0.2701 & 4.3099 & 24.3760 \\
\hline & 3 & 3 & 0.0429 & 0.2947 & 1.1788 & 1.1359 & 0.2518 & 4.5111 & 27.4779 \\
\hline & 3 & 4 & 0.0385 & 0.2817 & 1.1661 & 1.1276 & 0.2432 & 4.6365 & 30.2883 \\
\hline & 4 & 1 & 0.0473 & 0.2845 & 1.0048 & 0.9575 & 0.2372 & 4.0367 & 21.2431 \\
\hline & 4 & 2 & 0.0373 & 0.2399 & 0.9104 & 0.8731 & 0.2026 & 4.3095 & 24.4075 \\
\hline & 4 & 3 & 0.0321 & 0.2210 & 0.8841 & 0.8520 & 0.1889 & 4.5103 & 27.5421 \\
\hline & 4 & 4 & 0.0289 & 0.2113 & 0.8746 & 0.8457 & 0.1824 & 4.6365 & 30.2629 \\
\hline
\end{tabular}


Example 3: At $\alpha=0.05$ and $\beta=0.1$ let $\mu_{1}=0.01$ and $\mu_{2}=0.25$, then the operating ratio will be $\mu_{2} / \mu_{1}=25$. From Tab. 3 for this operating ratio, the nearer values are $24.4089,24.376$ and 24.4075. The nearest values of PQR and IQR corresponding to $s=3$ and $i=2$ will be found based on the minimum number of groups $g$.

Hence from Tab. 4, through quality region the required plan has parameters $s=3, g=5$ and $r=3$. The nearest values of PQR and IQR are $R_{1}=0.2328, R_{0}=0.0540$ and $T=4.3099$ corresponding to $s=3, g=5, r=3, i=2$ and $\mu_{1}=0.0107$.

Table 4: For $s=3$ and $i=2$ and specified $r$ the values of PQR, IQR, $\mu_{2} / \mu_{1}$ and $T$

\begin{tabular}{lllllllll}
\hline $\boldsymbol{r}$ & $\boldsymbol{\mu}_{\mathbf{2}} / \boldsymbol{\mu}_{\mathbf{1}}$ & $\boldsymbol{g} \boldsymbol{\mu}_{\mathbf{1}}$ & $\boldsymbol{g}$ & $\boldsymbol{\mu}_{\mathbf{1}}$ & $\boldsymbol{\mu}_{\mathbf{2}}$ & $\boldsymbol{R}_{\mathbf{1}}$ & $\boldsymbol{R}_{\mathbf{0}}$ & $T$ \\
\hline 2 & 24.4089 & 0.0746 & 8 & 0.0093 & 0.2276 & 0.2183 & 0.0507 & 4.3097 \\
3 & 24.3760 & 0.0498 & 5 & 0.0100 & 0.2428 & 0.2328 & 0.0540 & 4.3099 \\
4 & 24.4075 & 0.0373 & 4 & 0.0093 & 0.2276 & 0.2183 & 0.0507 & 4.3095 \\
\hline
\end{tabular}

\section{Conclusion}

In this acceptance sampling plan, both risks are used to balance the expected losses for the consumers and producers. The presented work is limited to BGChSP and estimate PQR and IQR for the specified producer's and consumer's risks. This research gives the idea to estimate an interval of average number of defectives for prespecified design parameters. Quality interval sampling plans have wider potential applications in the industry to ensure that the product or process complies with a higher quality standard. Thus, quality interval sampling could be useful for outlining product quality, planning, and quality control arrangements that are ready for electronic industrial applications. Many electronic components such as transport electronics systems, wireless systems, global positioning systems, and computer-supported and integrated manufacturing systems can be evaluated by using the proposed plan. Many other distributions and other quality and reliability characteristics can be explored in the future.

Funding Statement: This research was supported by the Ministry of Education (MOE) through Fundamental Research Grant Scheme (FRGS/1/2020/STG06/UUM/02/2), S/O Code 14884.

Conflicts of Interest: The authors declare that they have no conflicts of interest to report regarding the present study.

\section{References}

[1] M. Aslam, "A variable acceptance sampling plan under neuromorphic statistical interval method," Symmetry, vol. 11, no. 1, pp. 114, 2019.

[2] P. C. Ramyamol and M. Kumar, "Optimal reliability acceptance sampling plan based on accelerated life test data," International Journal of Productivity and Quality Management, vol. 30, no. 3, pp. 354$370,2020$.

[3] V. Gorgin, B. S. Gildeh, M. Sarmad and M. Amini, "On bivariate control charts for the mean of autocorrelated process," International Journal of Productivity and Quality Management, vol. 32, no. 1, pp. 72-91, 2021.

[4] S. A. Dobbah, M. Aslam and K. Khan, "Design of a new synthetic acceptance sampling plan," Symmetry, vol. 10, no. 11, pp. 653, 2018. 
[5] M. Aslam and A. H. Al-Marshadi, "Design of sampling plan using regression estimator under indeterminacy," Symmetry, vol. 10, no. 12, pp. 754, 2018.

[6] K. P. Veerakumari and S. Suganya, "Characteristic evaluation of SkSP-T with special type double sampling plan as reference plan," International Journal of Productivity and Quality Management, vol. 28, no. 3, pp. 360-371, 2019.

[7] W. T. Huang and Y. P. Lin, "An improved Bayesian sampling plan for exponential population with type I censoring," Communications in Statistics-Theory and Methods, vol. 31, no. 11, pp. 2003-2025, 2002.

[8] M. S. F. Nezhad and S. Seifi, "Designing optimal double-sampling plan based on process capability index," Communications in Statistics-Theory and Methods, vol. 46, no. 13, pp. 6624-6634, 2017.

[9] B. Epstein, "Truncated life tests in the exponential case," The Annals of Mathematical Statistics, vol. 25, no. 3, pp. 555-564, 1954.

[10] H. F. Dodge, "Chain sampling plan," Industrial Quality Control, vol. 11, pp. 10-13, 1955.

[11] H. F. Dodge and K. S. Stephens, A general family of chain sampling inspection plans. NJ: Rutgers-The State University New Brunswick, 1964.

[12] A. Hald, "Bayesian single sampling plans for discrete prior distribution," Mat. Fys. Skr. Dan. Vid. Selsk., vol. 3, no. 2, pp. 88, 1965 .

[13] K. Subbiah and M. Latha, "Selection of Bayesian single sampling plan with weighted Poisson distribution based on (AQL, LQL)," International Journal of Engineering Technology, vol. 5, no. 6, pp. 122134, 2017.

[14] C. Raju and K. K. Vidya, "Chain sampling plan (ChSP-1) for desired acceptable. Quality level (AQL) and limiting quality level (LQL)," in AIP Conf. Proc., AIP Publishing LLC, vol. 1905, pp. 50036, 2017.

[15] M. Latha and K. K. Suresh, "Construction and evaluation of performance measures for Bayesian chain sampling plan (BChSP-1)," For East Journal of Theoretical Statistics, vol. 6, no. 2, pp. 129-139, 2002.

[16] M. Latha and R. Arivazhagan, "Selection of Bayesian double sampling plan based on beta prior distribution index through quality region," International Journal of Recent Scientific Research, vol. 6, no. 5, pp. 4328-4333, 2015.

[17] M. C. Mathew and R. Murugesan, "Two sided complete Bayesian chain sampling plan with gamma Poisson as prior distribution," International Journal of Pure and Applied Mathematics, vol. 18, no. 5, pp. 207-213, 2018.

[18] M. Aslam and C. H. Jun, "A group acceptance sampling plan for truncated life test having Weibull distribution," Journal of Applied Statistics, vol. 36, no. 9, pp. 1021-1027, 2009.

[19] M. Aslam, A. R. Mughal, M. Ahmad and Z. Yab, "Group acceptance sampling plans for Pareto distribution of the second kind," Journal of Testing and Evaluation, vol. 38, no. 2, pp. 143-150, 2010.

[20] A. R. Mughal and M. Aslam, "Efficient group acceptance sampling plans for family Pareto distribution," Continental Journal of Applied Sciences, vol. 6, no. 3, pp. 40-52, 2011.

[21] A. R. Mughal and M. Ismail, "An economic reliability efficient group acceptance sampling plans for family Pareto distributions," Res. J. Appl. Sci., Eng. and Tech., vol. 6, no. 24, pp. 4646-4652, 2013.

[22] A. R. Mughal, Z. Zain and N. Aziz, "Economic reliability GASP for pareto distribution of the 2nd kind using poisson and weighted poisson distribution," Research Journal of Applied Sciences, vol. 10, no. 8, pp. 306-310, 2015.

[23] A. R. Mughal, Z. Zain and N. Aziz, "Time truncated group chain sampling strategy for pareto distribution of the 2(nd) Kind," Research Journal of Applied Sciences Engineering and Technology, vol. 10, no. 4, pp. 471-474, 2015.

[24] A. R. Mughal, "A family of group chain acceptance sampling plans based on truncated life test," Ph.D. dissertation. Universiti Utara Malaysia, Malaysia, 2018.

[25] W. Hafeez and N. Aziz, "Bayesian group chain sampling plan based on beta binomial distribution through quality region," International Journal of Supply Chain Management, vol. 8, no. 6, pp. 11751180, 2019. 
[26] K. K. Suresh and V. Sangeetha, "Construction and selection of Bayesian chain sampling plan (BChSP1) using quality regions,” Modern Applied Science, vol. 5, no. 2, pp. 226-234, 2011.

[27] K. M. Hamdia, M. A. Msekh, M. Silani, T. Q. Thai, P. R. Budarapu et al., "Assessment of computational fracture models using Bayesian method," Engineering Fracture Mechanics, vol. 205, no. 5, pp. 387-398, 2019. 